\title{
TEM Characterization of ZnO Nanoparticles Obtained by Mechanosynthesis
}

\author{
H.J. Morales Rodríguez, F. Espinosa-Magaña
}

Centro de Investigación en Materiales Avanzados, S.C., Laboratorio Nacional de Nanotecnología. Miguel de Cervantes 120, Chihuaua, Chih., México 31109

$\mathrm{ZnO}$ is a wide-band-gap semiconductor $(3.4 \mathrm{eV})$, which has attracted considerable attention during the last two decades due to its potential applications in optics and optoelectronics. $\mathrm{ZnO}$ has been used for short-wavelength laser devices and light-emitting diodes [1], and more recently, researchers have reported its possible application in solar cells [2],[3],[4]. $\mathrm{ZnO}$ has been used as a diluted magnetic semiconductor, when doped with transition metals and even pure nanostructured $\mathrm{ZnO}$ has been found to present magnetic behavior [5].

Using commercial $\mathrm{ZnO}$ powders as a starting point, particle size was reduced by mechanical milling in a high energy shaker SPEX mill. The apparatus and milling media are made of hardened steel and the milling ball to powder weight ratio was set to 5:1

Thin specimens suitable for electron microscopy were prepared by placing clean, dry crushed powders onto commercial holey carbon coated copper grids. Morphology and particle size was determined in a JEOL-2200FS HR-FE-TEM, equipped with an in-column energy filter ( $\Omega$-filter), with a spatial resolution of about $0.16 \mathrm{~nm}$.

Furthermore, Electron Energy Loss Spectroscopy (EELS) was used to monitor changes in electronic structure of $\mathrm{ZnO}$ as particle size decreases. The energy resolution of the EELS spectra was determined by measuring the full width at half-maximum (FWHM) of the zero loss peak, which was typically close to $1.0 \mathrm{eV}$ when the TEM was operated at $200 \mathrm{kV}$. EELS spectra were Fourier-Log deconvoluted to obtain the single scattering distributions $\mathrm{S}(\mathrm{E})$ and the real and imaginary parts of the dielectric function were obtained, after removing surface loss effects, by Kramers-Kronig Analysis.

Figs. 1-3 show TEM micrographs of $\mathrm{ZnO}$ particles after 0, 10 and $15 \mathrm{hrs}$. milling times, respectively. Fig. 4 shows the Kramers-Kronig derived imaginary part of the dielectric function, $\varepsilon_{2}$, from EELS spectra, for three particle sizes studied $(200,100,20 \mathrm{~nm})$, and corresponding to 0,10 and $15 \mathrm{hr}$ milling times, respectively. Spectra were shifted up for clarity. In all three spectra, five well defined peaks can be identified, labeled as A, B, C, D and E. Energy peak position A cannot be stated accurately, due to the uncertainties introduced by the zero-loss peak removal. Peak B is found at 8.8 $\mathrm{eV}$ and does not seem to shift as particle size is changed. Featured peak $\mathrm{C}$ shifts monotonically to lower energy as particle size decreases: $12.6,12.4$ and $12.1 \mathrm{eV}$ for 200,100 and $20 \mathrm{~nm}$, respectively. Featured peak D appears as a shoulder whose actual energy position cannot be stated unambiguously because of energy resolution limitations. Peak E remains constant at $31.3 \mathrm{eV}$ for 200 and $100 \mathrm{~nm}$ particle size, but decreases to $29.7 \mathrm{eV}$ for $20 \mathrm{~nm}$ particles.

\section{References}

[1] Hamdani, F., Botchkarev, A.E., Tang, H., Kim, W., and Morkoc, H. Appl. Phys. Lett. 71 (1997) 3111 .

[2] Keis, K., Vayssieres, V., Lindquist, S., and Hagfeldt, A.,. NanoStructured Materials, 12 (1999) 487.

[3] Shirakata, S., Takemi, T., Awai, K., Yamamoto, T. Thin Solid Films, 451-452 (2004) 212.

[4] Guillén, E., Casanueva, F., Anta, J.A., Vega-Poot, A., Oskam, G., Alcántara, R., FernándezLorenzo, C., Martín-Calleja, J., J. Photochemistry and Photobiology A: Chemistry 200 (2008) 364.

[5] Escudero, R., Escamilla, R., Solid State Commun. 151, (2011) 997. 


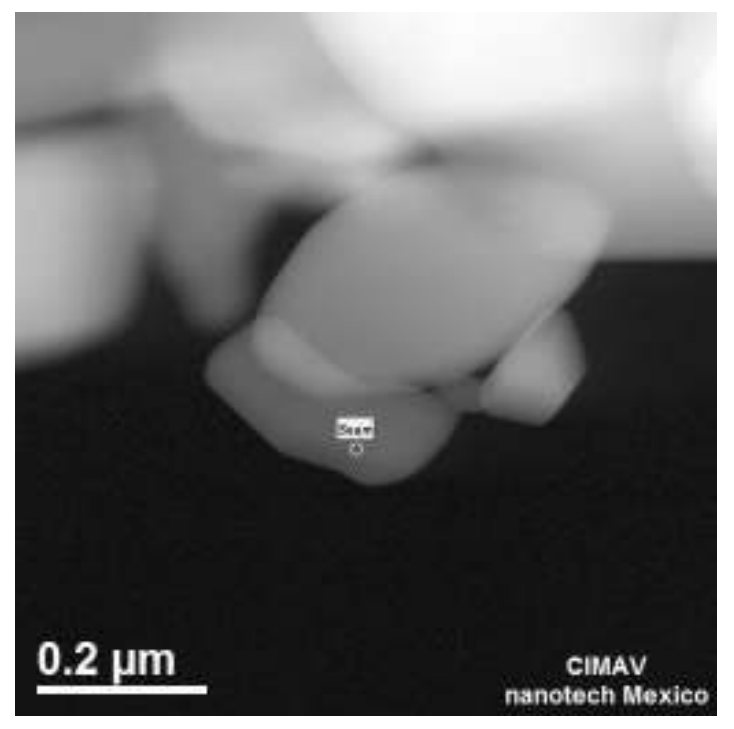

Figure 1. TEM micrograph of commercial $\mathrm{ZnO}$

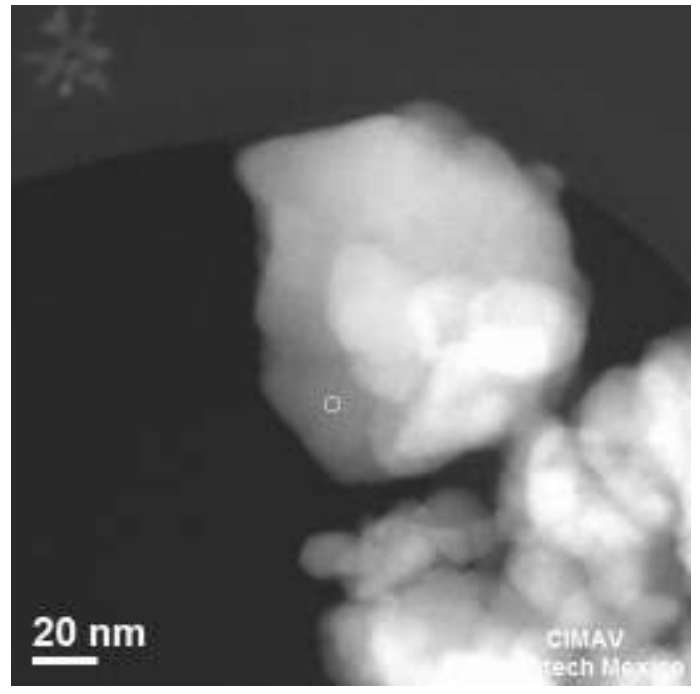

Figure 3. TEM micrograph of $\mathrm{ZnO}$ after 15 hrs. milling

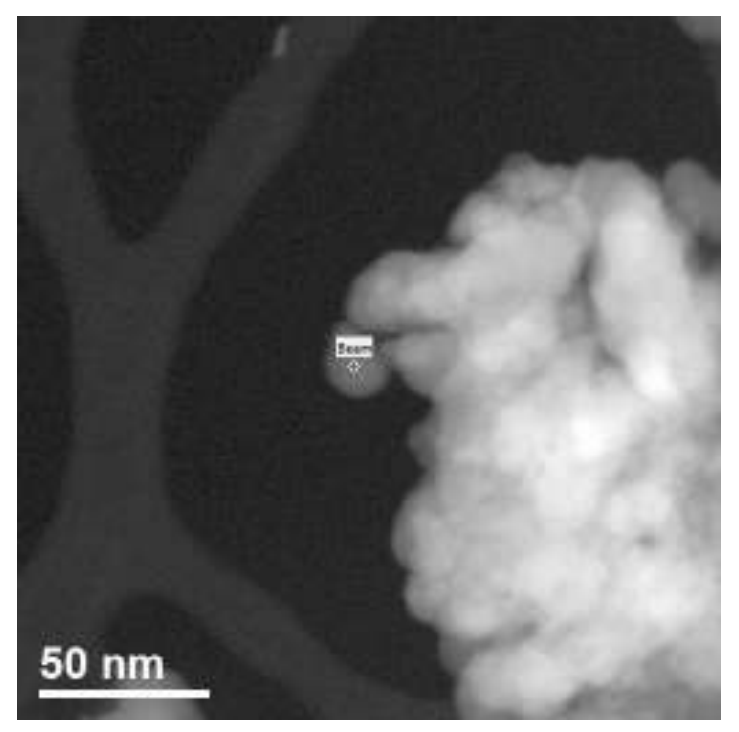

Figure 2. TEM micrograph of $\mathrm{ZnO}$ after 10 hrs. milling

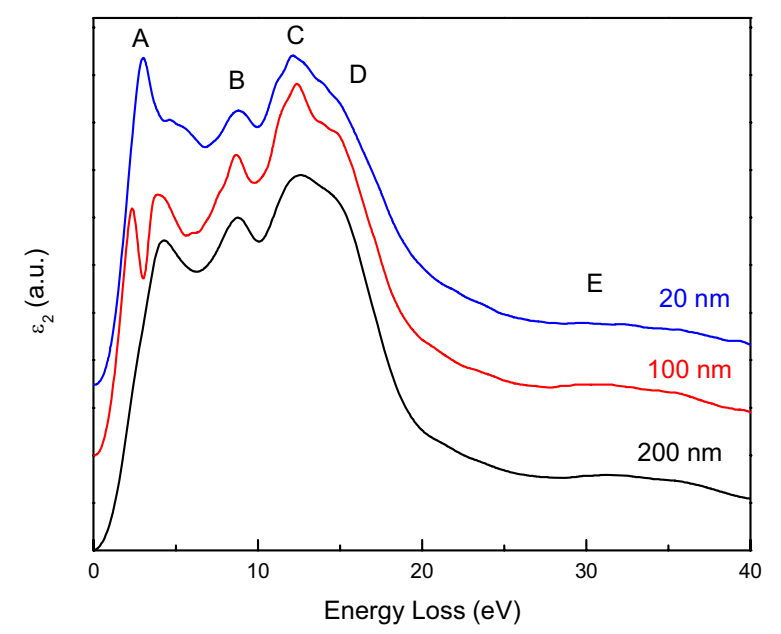

Figure 4. Kramers-Kronig derived imaginary part of the dielectric function vs. particle size 